\title{
On a New Class of Regular Doubly Stochastic Processes
}

\author{
Reza Farhadian", Nader Asadian \\ Department of Mathematics and Statistics, Lorestan University, Khorramabad, Lorestan, Iran
}

Email address:

farhadian.reza@yahoo.com (R. Farhadian), asadian.n@lu.ac.ir (N. Asadian)

*Corresponding author

\section{To cite this article:}

Reza Farhadian, Nader Asadian. On a New Class of Regular Doubly Stochastic Processes. American Journal of Theoretical and Applied Statistics. Vol. 6, No. 3, 2017, pp. 156-160. doi: 10.11648/j.ajtas.20170603.14

Received: March 17, 2017; Accepted: March 29, 2017; Published: May 25, 2017

\begin{abstract}
In this article, we show that the well-known Helmert matrix has strong relationship with stochastic matrices in modern probability theory. In fact, we show that we can construct some stochastic matrices by the Helmert matrix. Hence, we introduce a new class of regular and doubly stochastic matrices by use of the Helmert matrix and a special diagonal matrix that is defined in this article. Afterwards, we obtain the stationary distribution for this new class of stochastic matrices.
\end{abstract}

Keywords: Helmert Matrix, Stochastic Matrix, Markov Chain, Transition Probability, Stationary Distribution, Regular Chain, Ergodic Chain

\section{Introduction}

In modern probability theory and dynamical systems, stochastic processes and Markov chains are applied contexts that are used in advanced sciences. Two basic topics in stochastic process are prediction and filtering. In the topic of prediction for Markov chains, we can obtain the $k$-step transition probability, using the 1-step transition probability. This work is done by a matrix which is called the stochastic or probability or transition or Markov matrix. In stochastic processes or Markov chains, stochastic matrices are used for showing the transition probabilities [9, 11]. On the other hand, there is a special matrix in liner algebra that is called the Helmert matrix. A Helmert matrix of $\operatorname{order} n$ is a square matrix that was introduced by H. O. Lancaster in 1965 [4]. Usually, the Helmert matrix is used in mathematical statistics for analysis of variance (ANOVA), see [1, 2, 8]. In this article, we will show that the Helmert matrix can be used in stochastic processes. For the next sections, the following notation will be used:

(a) $I_{n}$ denotes an identity matrix of order $n$.

(b) $J_{n}$ denotes an $n \times n$ matrix whose elements are all 1 .

(c) $A_{n}{ }^{-1}$ denotes the inverse of a matrix $A_{n}$.

(d) $A_{n}{ }^{T}$ denotes the transpose of a matrix $A_{n}$.

(e) $\operatorname{Diag}\left[\begin{array}{llll}a_{11} & a_{22} & \ldots & a_{n n}\end{array}\right]$ denotes an $n \times n$ diagonal matrix with diagonal entries $a_{11}, a_{22}, \ldots, a_{n n}$.

(f) $A_{n}>0$ stands for a matrix $A_{n}$ all of whose elements are positive. (g) $\mathbb{R}$ denotes real numbers.

\section{Definitions and Particulars}

\subsection{Stochastic Matrix}

Suppose that a stochastic process start from state $i$ to state $j$. This transition shown by $i \rightarrow j$, and $p_{i j}$ denote its probability. Now, if process consist of $n$ states and $X_{t}$ denotes the state at time $t$, then the transition $i \rightarrow j$ at time $t$, is indicated by $X_{t}=i$ and $X_{t+1}=j$. Furthermore, a process is called a Markov chain if the transition probability $p_{i j}$ is independent of time $t$ for every states $i$ and $j$ of state space. Hence, the transition probability under the Markov property, is defined as:

$$
\begin{aligned}
p_{i j}=\operatorname{Pr}\left\{X_{t+1}\right. & \left.=j \mid X_{t}=i, X_{t-1}=i_{t-1}, \ldots, X_{1}=i_{1}\right\} \\
& =\operatorname{Pr}\left\{X_{t+1}=j \mid X_{t}=i\right\}
\end{aligned}
$$

for every $i_{1}, i_{2}, \ldots, i_{t-1}, i, j$ of state space. Thus, we can construct a $n \times n$ stochastic (transition) matrix by the $p_{i j}$, $1 \leq i, j \leq n$ :

Definition 1. [9] $A n \times n$ real matrix such as $P_{n}=$ $\left[p_{i j}\right]_{n \times n}$ is called a stochastic matrix (or row stochastic matrix), if

1) $p_{i j} \geq 0,1 \leq i, j \leq n$ 
2) $\sum_{j=1}^{n} p_{i j}=1, \forall i \in\{1,2, \ldots, n\}$

Definition 2. [5] A doubly stochastic matrix, is a square matrix of nonnegative real numbers with each row and column summing to 1(in other words, a stochastic matrix is doubly, if its transpose is stochastic matrix).

Hence, If $\left[p_{i j}\right]_{n \times n}$ be the 1-step transition matrix, then the matrix $\left[p_{i j}{ }^{(k)}\right]_{n \times n}$ is called the $k$-step transition matrix (under the conditions of Definition 1). Chapman and Kolmogorov independently showed that if $P$ is a 1-step transition matrix, then $k$-step transition matrix denoted by $P^{(k)}$ and is equal to

$$
P^{(k)}=P^{k}=\underbrace{P \times P \times \ldots \times P}_{k \text { times }}
$$

Or in other words, if $\ell$ be the state space of a Markov chain, then

$$
p_{i j}^{(k)}=\operatorname{Pr}\left\{X_{t+k}=j \mid X_{t}=i\right\}=\sum_{z \in \ell} p_{i z}^{(r)} p_{z j}^{(k-r)}
$$

where $0<r<k$. For more details about the ChapmanKolmogorov equation, see $[9,10,11]$.

\subsection{Regular and Ergodic Markov Chains and Stationary Distribution}

A nonnegative square matrix $P$ is called regular if $P^{m}>0$ for some $m$ [6]. Since every transition matrix is nonnegative, hence we have a similar definition for Markov chains.

Definition 3. [7] A Markov chain is called a regular chain if some powers of the transition matrix has only positive elements (in other words, the transition matrix of chain be regular).

Regularity is an important property for Markov chains since it has a strong relationship with another important ergodicity property. We know that a Markov chain is ergodic if it is possible to go from every state to every state (not necessarily in one move). By [3, Theorem 1.8] we know that if a Markov transition matrix $P$ is regular, then it has exactly one ergodic class and in general this process is ergodic. Hence, the following proposition shows the relationship between the regularity property and ergodicity one:

Proposition 1. [3, 6] Every regular Markov chain is ergodic.

Ergodic Markov chains are very important because there is a unique stationary distribution vector for their states. To compute the stationary distribution of an ergodic Markov chain, we have:

Proposition 2. [9] Let $p_{i j}{ }^{(k)}$ be the $k$-step transition probability from state $i$ to statej and $\lim _{k \rightarrow \infty} p_{i j}{ }^{(k)}$ exists. If $\pi_{j}$ denotes the stationary distribution and chain be ergodic, then $\pi_{j}=\lim _{k \rightarrow \infty} p_{i j}{ }^{(k)}$.

Since every regular chain is ergodic, if chain be regular, then stationary distribution exists.

\subsection{The Helmert Matrix}

The Helmert matrix is a square matrix of order $n$ that is defined as:

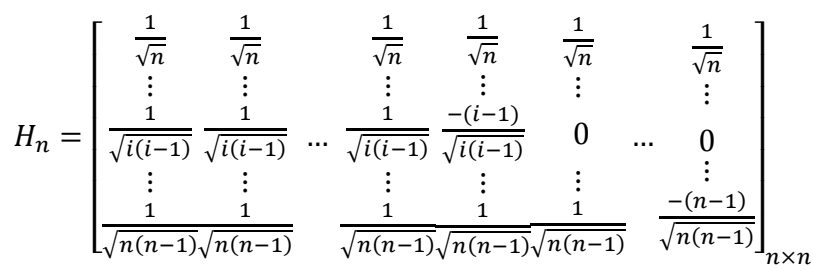

Also see [2, page 67].

Moreover, the first row of the Helmert matrix of order $n$, has the following form

$$
\underbrace{\left[\begin{array}{lllll}
\frac{1}{\sqrt{n}} & \frac{1}{\sqrt{n}} & \frac{1}{\sqrt{n}} & \cdots & \frac{1}{\sqrt{n}}
\end{array}\right]}_{n \text { items }}
$$

And the other $i$-th rows $(2 \leq i \leq n)$ are formed by

$$
\left[\begin{array}{llllll}
\frac{1}{\sqrt{(i-1) i}} \frac{1}{\sqrt{(i-1) i}} & \cdots & \frac{1}{\sqrt{(i-1) i}} & \frac{-(i-1)}{\sqrt{(i-1) i}} & \underbrace{0}_{n-i \text { items }} \quad \ldots \quad 0
\end{array}\right]
$$

Furthermore, we know that the Helmert matrix is orthogonal [1]:

$$
H_{n} H_{n}{ }^{T}=H_{n}{ }^{T} H_{n}=I_{n}
$$

To prove the main theorems in the next section, we need the following proposition:

Proposition 3. Let $H_{n}$ be the Helmert matrix of order $n$ and $D_{n}=\operatorname{Diag}[\gamma+n \beta \quad \gamma \quad \gamma \quad \ldots \quad \gamma]$ with $\gamma, \beta \in \mathbb{R}$. Then

$$
H_{n}{ }^{T} D_{n} H_{n}=\gamma I_{n}+\beta J_{n}
$$

Proof. By calculation we have

$$
H_{n}{ }^{T} \times D_{n}=\gamma \times H_{n}{ }^{T}+\left[\begin{array}{cccc}
\frac{n \beta}{\sqrt{n}} & 0 & \ldots & 0 \\
\frac{n \beta}{\sqrt{n}} & 0 & \ldots & 0 \\
\vdots & \vdots & \ddots & \vdots \\
\frac{n \beta}{\sqrt{n}} & 0 & \ldots & 0
\end{array}\right]_{n \times n}
$$

Besides, by calculation, we have

$$
\beta \times J_{n} \times H_{n}{ }^{T}=\left[\begin{array}{llll}
\frac{n \beta}{\sqrt{n}} & 0 & \ldots & 0 \\
\frac{n \beta}{\sqrt{n}} & 0 & \ldots & 0 \\
\vdots & \vdots & \ddots & \vdots \\
\frac{n \beta}{\sqrt{n}} & 0 & \ldots & 0
\end{array}\right]_{n \times n}
$$

Hence, using (10) in (9), we obtain

$$
H_{n}{ }^{T} \times D_{n}=\gamma \times H_{n}{ }^{T}+\beta \times J_{n} \times H_{n}{ }^{T}
$$

Right multiplying of both sides of (11) by $H_{n}$, we obtain

$$
H_{n}{ }^{T} \times D_{n} \times H_{n}=\gamma \times I_{n}+\beta \times J_{n}
$$

The theorem is proved. 


\section{Main Results}

\subsection{A New Class of Regular Doubly Stochastic Matrices Using the Helmert Matrix}

Let us consider the matrix

$$
P_{n}=H_{n}{ }^{T} D_{n} H_{n}
$$

where $H_{n}$ is the Helmert matrix of order $n$ and $D_{n}=$ $\operatorname{Diag}\left[\begin{array}{lllll}1 & \frac{a}{a+n b} & \frac{a}{a+n b} & \cdots & \frac{a}{a+n b}\end{array}\right]$ with $a, b \geq 0$. Clearly, the matrix $P_{n}$ is a diagonalizable matrix (note that in matrix theory, we know that if a square matrix of order $n$ such as $A_{n}$ can be equal to $B_{n}{ }^{T} D_{n} B_{n}$ where $B_{n}$ is a orthogonal matrix and $D_{n}$ is a diagonal matrix, then $A_{n}$ is called a diagonalizable matrix (see [12])).

Now, we shall prove that $P_{n}$ is a doubly stochastic matrix. We need the following lemma.

Lemma 2. Let $H_{n}$ be the Helmert matrix of order $n$ and $a, b \geq 0$ such that at least $a$ or $b$ is positive. Then

$$
H_{n}{ }^{T} D_{n} H_{n}=\frac{1}{a+n b}\left(a I_{n}+b J_{n}\right)
$$

where $D_{n}=\operatorname{Diag}\left[\begin{array}{lllll}1 & \frac{a}{a+n b} & \frac{a}{a+n b} & \cdots & \frac{a}{a+n b}\end{array}\right]$.

Proof. Using Proposition 3 with $\gamma=\frac{a}{a+n b}$ and $\beta=\frac{b}{a+n b}$, the lemma is proved.

Theorem 1. Let $D_{n}=\operatorname{Diag}\left[\begin{array}{lllll}1 & \frac{a}{a+n b} & \frac{a}{a+n b} & \cdots & \frac{a}{a+n b}\end{array}\right]$ for $a, b \geq 0$ (at least $a$ or $b$ is positive) and $H_{n}$ be the Helmert matrix of order $n$. Then $P_{n}=H_{n}{ }^{T} D_{n} H_{n}$ is a doubly stochastic matrix.

Proof. By Lemma 2, we know that $P_{n}=H_{n}{ }^{T} D_{n} H_{n}=$ $\frac{1}{a+n b}\left(a I_{n}+b J_{n}\right)$. So, we have

$$
\begin{aligned}
P_{n}=\frac{1}{a+n b}(a \overbrace{\left[\begin{array}{ccc}
1 & \cdots & 0 \\
\vdots & \ddots & \vdots \\
0 & \cdots & 1
\end{array}\right]_{n \times n}}^{I_{n}}+b \overbrace{\left[\begin{array}{ccc}
1 & \cdots & 1 \\
\vdots & \ddots & \vdots \\
1 & \cdots & 1
\end{array}\right]_{n \times n}}^{J_{n}}) \\
= \\
=\left[\begin{array}{cccc}
\frac{a+b}{a+n b} & \frac{b}{a+n b} & \cdots & \frac{b}{a+n b} \\
\frac{b}{a+n b} & \frac{a+b}{a+n b} & \cdots & \frac{b}{a+n b} \\
\vdots & \vdots & \ddots & \vdots \\
\frac{b}{a+n b} & \frac{b}{a+n b} & \cdots & \frac{a+b}{a+n b}
\end{array}\right]_{n \times n}
\end{aligned}
$$

Clearly, all elements of the above matrix are positive. Now, we shall prove that each row of (14) sum to 1 . Hence, we have

For the 1-th row: $\frac{a+b}{a+n b}+\underbrace{\frac{b}{a+n b}+\frac{b}{a+n b}+\cdots+\frac{b}{a+n b}}=\frac{a+n b}{a+n b}=1$

For the 2-th row: $\frac{b}{a+n b}+\frac{a+b}{a+n b}+\underbrace{\frac{n-1 \text { items }}{a+n b}+\cdots+\frac{b}{a+n b}}_{n-2 \text { items }}=\frac{a+n b}{a+n b}=1$

For the $i$-th row:

$$
\begin{gathered}
\underbrace{\frac{b}{a+n b}+\frac{b}{a+n b}+\cdots+\frac{b}{a+n b}}+\frac{a+b}{a+n b}+ \\
\underbrace{\frac{b}{a+n b}+\frac{b}{a+n b}+\cdots+\frac{b}{a+n b}}_{n \text {-iitems }}=(n-1) \frac{b}{a+n b}+\frac{a+b}{a+n b}=\frac{a+n b}{a+n b}=1
\end{gathered}
$$

For the $n$-th row: $\underbrace{\frac{b}{a+n b}+\frac{b}{a+n b}+\cdots+\frac{b}{a+n b}}_{n-1 \text { items }}+\frac{a+b}{a+n b}=\frac{a+n b}{a+n b}=1$

So, both conditions of Definition 1 are holds for the matrix $P_{n}=H_{n}{ }^{T} D_{n} H_{n}$, hence this matrix is a stochastic matrix.

On the other hand, we know that the matrix $P_{n}$ is a symmetric matrix, because

$$
P_{n}{ }^{T}=\left(H_{n}{ }^{T} D_{n} H_{n}\right)^{T}=H_{n}{ }^{T} D_{n} H_{n}=P_{n}
$$

Thus, by Definition 2, it is a doubly stochastic matrix.

Corollary 1. Let $\left\{X_{t}: t \geq 0\right\}$ be the finite Markov chain by stochastic matrix $P_{n}$ in Theorem 1. Then the transition probability for transition $i \rightarrow j$ is equal to

$$
p_{i j}=\operatorname{Pr}\left\{X_{t+1}=j \mid X_{t}=i\right\}= \begin{cases}\frac{a+b}{a+n b} & i=j \\ \frac{b}{a+n b} & i \neq j\end{cases}
$$

Proof. We know that $p_{i j}$ is the $(i, j)$-th element of stochastic matrix $P_{n}$. Therefore the proof is immediately.

Suppose that a square $n \times n$ doubly stochastic matrix such as $Q_{n}$ has the following form

$$
Q_{n}=\left[\begin{array}{cccc}
q_{11} & q_{12} & \ldots & q_{1 n} \\
q_{21} & q_{22} & \ldots & q_{2 n} \\
\vdots & \vdots & \ddots & \vdots \\
q_{n 1} & q_{n 2} & \ldots & q_{n n}
\end{array}\right]_{n \times n}=\left[\begin{array}{cccc}
s & t & \ldots & t \\
t & s & \ldots & t \\
\vdots & \vdots & \ddots & \vdots \\
t & t & \ldots & s
\end{array}\right]_{n \times n}
$$

Clearly, since $q_{i i}=s$ and $q_{i j}=t$ (for $i \neq j$ ), then the matrix $Q_{n}$ is follow of new class, if $s \geq t$ and the following system of equations hold:

$\left\{\begin{array}{l}s=\frac{a+b}{a+n b} \\ t=\frac{b}{a+n b}\end{array} \Rightarrow a, b \geq 0\right.$ and at least $a$ or $b$ is positive

Hence we can write $Q_{n}=\frac{1}{a+n b}\left(a I_{n}+b J_{n}\right)$.

Example 1. Consider the matrix $Q_{3}=\left[\begin{array}{ccc}\frac{3}{7} & \frac{2}{7} & \frac{2}{7} \\ \frac{2}{7} & \frac{3}{7} & \frac{2}{7} \\ \frac{2}{7} & \frac{2}{7} & \frac{3}{7}\end{array}\right]_{3 \times 3}$. We can show that $Q_{3}$ is a doubly stochastic matrix and follow of the new class. Since if it is considered $=\frac{3}{7}, t=\frac{2}{7}$ and $n=3$, then by (18) we have

$$
\left\{\begin{array}{l}
\frac{3}{7}=\frac{a+b}{a+3 b} \\
\frac{2}{7}=\frac{b}{a+3 b}
\end{array} \Rightarrow a=1, b=2\right.
$$

Since both $a=1$ and $b=2$ are positive, so, by (18) the matrix $Q_{3}$ is follow of the new class. 
Now, consider the following another example.

Example 2. Let $Q_{2}=\left[\begin{array}{cc}\frac{2}{5} & \frac{3}{5} \\ \frac{3}{5} & \frac{2}{5}\end{array}\right]_{2 \times 2}$. Clearly, $Q_{2}$ is a doubly stochastic matrix. But $Q_{2}$ is not follow of new class, because if $s=\frac{2}{5}$ and $t=\frac{3}{5}$, then we know that $s<t$, and this is in contradiction with (17) and (18).

Corollary 2. Let $Q_{n}$ is a doubly stochastic matrix such that it is hold under the (18). Then $Q_{n}$ is diagonalizable and $Q_{n}=H_{n}{ }^{T} D_{n} H_{n}$ where $H_{n}$ is the Helmert matrix of order $n$ and $D_{n}=\operatorname{Diag}\left[\begin{array}{lllll}1 & \frac{a}{a+n b} & \frac{a}{a+n b} & \cdots & \frac{a}{a+n b}\end{array}\right]$

Proof. By Corollary 1 if (18) holds for the matrix $Q_{n}$, then $Q_{n}$ is follow of the new class and $Q_{n}=\frac{1}{a+n b}\left(a I_{n}+b J_{n}\right)$. On the other hand, by Lemma 2, we know that the matrix $Q_{n}=\frac{1}{a+n b}\left(a I_{n}+b J_{n}\right)$ is diagonalizable and we can write $Q_{n}=\frac{1}{a+n b}\left(a I_{n}+b J_{n}\right)=H_{n}{ }^{T} D_{n} H_{n} \quad$ where $\quad H_{n} \quad$ is the Helmert matrix of order $n$ and $D_{n}$ is the same diagonal matrix in Lemma 2.

When a matrix is diagonalizable, we can obtain its positive integer powers by its diagonal form. On the other hand, by Chapman-Kolmogorov equation $[9,10,11]$, we know that the $k$-step transition matrix is equal to the $k$-th power of 1-step transition matrix. Hence, for the stochastic matrix $Q_{n}$ in Corollary 2 , we have

$$
Q_{n}{ }^{k}=H_{n}{ }^{T} D_{n}{ }^{k} H_{n}
$$

In the next part, we will use of (19) to compute the stationary distribution of the new class of stochastic matrices.

\subsection{Stationary Distribution for the New Class of Regular Stochastic Matrices}

We know that the Markov chain by stochastic matrix in the Theorem 1, is a regular and ergodic chain, since all elements in this matrix are positive and hence is possible to go from every state to every state. Now, in the next theorem we compute the stationary distribution for this chain.

Lemma 3. Let $H_{n}$ be the Helmert matrix of order $n$ and $D_{n}=\operatorname{Diag}\left[\begin{array}{lllll}1 & 0 & 0 & \ldots & 0\end{array}\right]$. Then $H_{n}{ }^{T} D_{n} H_{n}=\frac{1}{n} J_{n}$.

Proof. The lemma is proved by Lemma 2 with $a=0$.

Theorem 2. The Markov chain by the stochastic matrix in Theorem 1 is a regular and ergodic chain, and if $\pi_{j}(j=1,2, \ldots, n)$ denotes the stationary distribution of this chain, then $\pi_{j}=\frac{1}{n}$.

Proof. By Theorem 1 we know that all elements of stochastic matrix $P_{n}$ are positive. So, the chain is regular. Besides, by Proposition 1, the chain must be ergodic. By Proposition 2, we know that if chain is ergodic and $\lim _{k \rightarrow \infty} p_{i j}{ }^{(k)}$ exists, then $\pi_{j}=\lim _{k \rightarrow \infty} p_{i j}{ }^{(k)}$. So, we have

$$
\operatorname{Lim}_{k \rightarrow \infty} P_{n}{ }^{k}=\lim _{k \rightarrow \infty}\left(H_{n}{ }^{T} D_{n} H_{n}\right)^{k}
$$

We know that $D_{n}=\operatorname{Diag}\left[\begin{array}{lllll}1 & \frac{a}{a+n b} & \frac{a}{a+n b} & \cdots & \frac{a}{a+n b}\end{array}\right]$. Using (19), we have

$$
\begin{aligned}
& \lim _{k \rightarrow \infty}\left(H_{n}{ }^{T} \times\left[\begin{array}{cccc}
1 & & & \\
& \frac{a}{a+n b} & & 0 \\
& 0 & & \frac{a}{a+n b}
\end{array}\right]_{n \times n} \times H_{n}\right)^{k}
\end{aligned}
$$

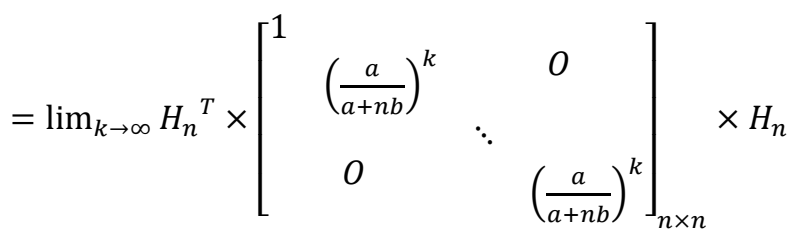

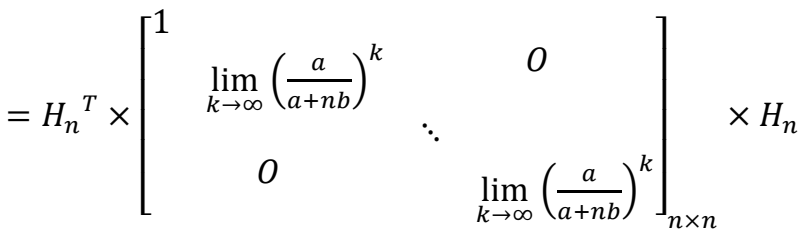

$$
\begin{aligned}
& =H_{n}{ }^{T} \times\left[\begin{array}{cccc}
1 & 0 & \ldots & 0 \\
0 & 0 & \ldots & 0 \\
\vdots & \vdots & \ddots & \vdots \\
0 & 0 & \ldots & 0
\end{array}\right]_{n \times n} \times H_{n}
\end{aligned}
$$

And by Lemma 3, we have

$$
H_{n}{ }^{T} \times\left[\begin{array}{cccc}
1 & 0 & \ldots & 0 \\
0 & 0 & \ldots & 0 \\
\vdots & \vdots & \ddots & \vdots \\
0 & 0 & \ldots & 0
\end{array}\right]_{n \times n} \times H_{n}=\frac{1}{n} J_{n}
$$

Therefore $\pi_{j}=\frac{1}{n}$.

\section{Conclusion}

Usually, we can working on many topics of probability theory, but working on stochastic processes and offering new Markov chains are less than other subjects. In this article we presented a new class of stochastic matrices. Since for every integer $n$ and every positive real numbers such as $a$ and $b$, we can construct a stochastic matrix of order $n$ by forming to $P_{n}=\frac{1}{a+n b}\left(a I_{n}+b J_{n}\right)$, so the new class is very big. Also, we showed that $P_{n}=H_{n}{ }^{T} D_{n} H_{n}$ where $H_{n}$ is the well-known Helmert matrix and $D_{n}$ is a diagonal matrix of order $n$. So, the stochastic matrix $P_{n}$ is a diagonalizable matrix. In matrix theory, diagonalizable form of a square matrix is very important, because compute of determinant and also integer powers of matrix by this form is simpler than other usual methods. On the other hand, we know that integer powers of a stochastic matrix is important for prediction of its behavior. In addition, we showed that the Markov chain by the stochastic matrix $P_{n}$ is regular and ergodic. Regular and ergodic properties are two important properties of stochastic processes, because the stationary distribution for any regular or ergodic Markov chain is equal to the limit of $k$-th power of its transition matrix as $k \rightarrow \infty$. Furthermore, we proved that the stochastic matrix $P_{n}$ is a doubly stochastic matrix. 


\section{Appendix: Doubly Stochastic Matrices that Their Inverses are Doubly Stochastic Matrices}

We know that $I_{n}$ is a doubly stochastic matrix, since each row and column of $I_{n}$ sum to 1 . Besides, $I_{n}{ }^{-1}=I_{n}$, which means that there is at least a doubly stochastic matrix of order $n$ such that its inverse is a doubly stochastic matrix. In 2015, R. Farhadian (the first author) by a Farsi language article titled "Approximation for stationary distribution of ergodic stochastic processes" published in "NEDA: Student Statistical Journal", showed that there exists some doubly stochastic matrices except identity matrix, such that their inverses are doubly stochastic matrices. Consider the following matrices of order 2 and 3:

$$
M_{2}=\underbrace{\left[\begin{array}{cc}
\frac{1}{\sqrt{2}} & \frac{1}{\sqrt{2}} \\
\frac{1}{\sqrt{2}} & \frac{-1}{\sqrt{2}}
\end{array}\right]}_{\text {Diagonalized form }} \times\left[\begin{array}{cc}
1 & 0 \\
0 & -1
\end{array}\right] \times \overbrace{\left[\begin{array}{cc}
\frac{1}{\sqrt{2}} & \frac{1}{\sqrt{2}} \\
\frac{1}{\sqrt{2}} & \frac{-1}{\sqrt{2}}
\end{array}\right]}^{H_{2}}=\left[\begin{array}{ll}
0 & 1 \\
1 & 0
\end{array}\right]
$$

and

$$
\begin{gathered}
M_{3}=\overbrace{\left[\begin{array}{ccc}
\frac{1}{\sqrt{3}} & \frac{1}{\sqrt{3}} & \frac{1}{\sqrt{6}} \\
\frac{1}{\sqrt{3}} & \frac{-1}{\sqrt{2}} & \frac{1}{\sqrt{6}} \\
\frac{1}{\sqrt{3}} & 0 & \frac{-2}{\sqrt{6}}
\end{array}\right] \times\left[\begin{array}{ccc}
{ }^{T} & 0 & 0 \\
0 & -1 & 0 \\
0 & 0 & 1
\end{array}\right] \times \overbrace{\left[\begin{array}{ccc}
\frac{1}{\sqrt{3}} & \frac{1}{\sqrt{3}} & \frac{1}{\sqrt{3}} \\
\frac{1}{\sqrt{2}} & \frac{-1}{\sqrt{2}} & 0 \\
\frac{1}{\sqrt{6}} & \frac{1}{\sqrt{6}} & \frac{-2}{\sqrt{6}}
\end{array}\right]}^{H_{3}}}^{\text {Diagonalized form }} \\
=\left[\begin{array}{ccc}
0 & 1 & 0 \\
1 & 0 & 0 \\
0 & 0 & 1
\end{array}\right]
\end{gathered}
$$

where $\mathrm{H}_{2}$ and $\mathrm{H}_{3}$ are orthogonal Helmert matrices of order 2 and order 3 , respectively.

We know that for a diagonalizable square matrix such as $A_{n}=B_{n}{ }^{T} D_{n} B_{n}$, the inverse of $A_{n}$ is equal to $A_{n}=$ $B_{n}{ }^{T} D_{n}{ }^{-1} B_{n}$. Thus for inverses of $M_{2}$ and $M_{3}$, we have $M_{2}{ }^{-1}=\left[\begin{array}{cc}\frac{1}{\sqrt{2}} & \frac{1}{\sqrt{2}} \\ \frac{1}{\sqrt{2}} & \frac{-1}{\sqrt{2}}\end{array}\right] \times\left[\begin{array}{cc}(1)^{-1} & 0 \\ 0 & (-1)^{-1}\end{array}\right] \times\left[\begin{array}{cc}\frac{1}{\sqrt{2}} & \frac{1}{\sqrt{2}} \\ \frac{1}{\sqrt{2}} & \frac{-1}{\sqrt{2}}\end{array}\right]=\left[\begin{array}{ll}0 & 1 \\ 1 & 0\end{array}\right]$ and

$$
\begin{gathered}
M_{3}{ }^{-1}=\left[\begin{array}{ccc}
\frac{1}{\sqrt{3}} & \frac{1}{\sqrt{3}} & \frac{1}{\sqrt{6}} \\
\frac{1}{\sqrt{3}} & \frac{-1}{\sqrt{2}} & \frac{1}{\sqrt{6}} \\
\frac{1}{\sqrt{3}} & 0 & \frac{-2}{\sqrt{6}}
\end{array}\right] \times\left[\begin{array}{ccc}
(1)^{-1} & 0 & 0 \\
0 & (-1)^{-1} & 0 \\
0 & 0 & (1)^{-1}
\end{array}\right] \times \\
{\left[\begin{array}{ccc}
\frac{1}{\sqrt{3}} & \frac{1}{\sqrt{3}} & \frac{1}{\sqrt{3}} \\
\frac{1}{\sqrt{2}} & \frac{-1}{\sqrt{2}} & 0 \\
\frac{1}{\sqrt{6}} & \frac{1}{\sqrt{6}} & \frac{-2}{\sqrt{6}}
\end{array}\right]=\left[\begin{array}{lll}
0 & 1 & 0 \\
1 & 0 & 0 \\
0 & 0 & 1
\end{array}\right]}
\end{gathered}
$$

Clearly, $M_{2}$ and $M_{3}$ are doubly stochastic matrices and $M_{2}{ }^{-1}=M_{2}$ and $M_{3}{ }^{-1}=M_{3}$ are doubly stochastic matrices.

\section{References}

[1] B. R. Clarke. Linear models: the theory and application of analysis of variance. John Wiley \& Sons, New Jersey, 2008.

[2] J. E. Gentie, Numerical linear algebra for application in statistics, Springer, New York, 1998.

[3] A. Kehagias, Approximation of stochastic processes by hidden Markov models, U. M. I, 1992.

[4] H. O. Lancaster. The Helmert matrices. Amer. Math. Monthly, 72 (1965), no. 1, 4-12.

[5] E. Parzen, Stochastic processes, San Francisco: Holden-Day, Inc., 1962.

[6] P. Perkins, A theorem on regular matrices. Pacific J. Math. 11 (1961), no. 4, 1529-1533.

[7] Ch. M. Grinstead, J. L. Snell, Introduction to probability, American Mathematical Society, 1997.

[8] G. A. F. Seber, A matrix handbook for statistician, John Wiley \& Sons, New Jersey, 2007.

[9] S. M. Roos, A first cours in probability, $6^{\text {th }}$ ed, Pearson Prentice Hall, 2002.

[10] S. M. Ross, Introduction to probability models. $7^{\text {th }}$ ed. San Diego, colif: Academic Press, Inc., 2000.

[11] S. M. Ross, Stochastic processes. $2^{\text {nd }}$ ed. John wiley \& Sons, Inc., New York, 1996.

[12] X. Zhan, Matrix theory, Graduate studies in mathematics, volume 147, American Mathematical Society, 2013. 